\title{
Research of Dimensional Accuracy about Cone Case Double roller Spinning
}

\author{
Yu Yang \\ Changchun University of Science and Technology,Changchun,Jilin province,China \\ yangyu1983@sina.com
}

\begin{abstract}
Key words:shifty force; power spinning; diametric dimensional accuracy; surface roughness Abstract.For the computing method of conventional spinning force sometimes has a great error by neglecting deposit metal before roller, this essay proposed a spinning force calculation method based on cut and curving force, and it is analyzed an effective and accurate way by testing a set of craft experiments. Then setting up a model on the basis of this method and conducting the stimulation test about shifty force of the projectile two rollers power spinning. The experimental results demonstrate that this experimental method can effectively increase projectile in its diametric dimensional accuracy and decrease its surface roughness on condition.
\end{abstract}

\section{Introduction}

Spinning is a kind of chipless extrusion forming technology. The precision of the spinning products mainly comprises the parameters in two aspects: the precision of diameter of the spinning products and the surface roughness of the spinning products ${ }^{[1]}$. The precision of the spinning products is mainly affected by the feed rate; under the condition that other process parameters are kept constant, the increase of feed rate of spinning roller will cause rough surfaces of the spinning products. Otherwise, the reduction of the feed rate of spinning roller will not only lower the production rate, but cause the failure of profiling of the spinning products ${ }^{[2]}$. With a view to ensure the stable feed rate of the spinning roller, it is required to precisely determine the spinning force applied on the spinning roller in the process of spinning. At present, the evaluation of spinning force is conducted under the assumed condition that the metals are flowed axially in a stable and continuous manner; however, during actual spinning, the metal buildup caused due to the unsmooth flowing of metal will change the deformation rate of the spinning products and this is also the primary cause ${ }^{[3]}$ for the errors existed in the values of spinning force obtained respectively by the traditional algorithm and actual technical experiments. Just because of the inaccuracy of the calculated values, the average value of spinning force is mostly applied for the setting of spinning force in the process of spinning; but this will cause the oversized feed amount in the earlier stage of spinning process and the undersized feed amount in the latter stage of the process and meanwhile the molding quality of the spinning products will become unsatisfactory. Although the dimensional accuracy of the spinning products will be improved by applying the traditional algorithm for the setting of spinning force and power spinning of changing spinning force, the relatively larger errors in calculated values will cause the corrugation frequently occurring in the process of spinning and then it will take lots of time to carry out the adjustment of process parameters and relevant experiments of spinning process ${ }^{[4]}$.

This paper has put forward an algorithm which is based on the combined formation of shearing and bending moment to calculate spinning force. The calculation of spinning force of various stages in the process of machining is conducted to analyze the effect of deformation of metal materials on the machining precision. Meanwhile, the Electrometric Method is applied to measure the radial, axial and tangential spinning force exerted on the spinning roller in the power spinning of projectile bodies. The experimental results have proved the accuracy of the algorithm. The experiments on 3 kinds of spinning processes are carried out, including the power spinning by adopting the spinning force of fixed value, the power spinning in which the varied spinning forces by traditional algorithm are applied and the power spinning in which the varied spinning forces by new algorithm are applied. The experimental results show that the dimensional accuracy of projectile body spun by power spinning in which the varied spinning forces are determined by new algorithm is more obviously improved compared with that of the spinning products made by another two spinning 
processes. The result of the experiment is identical with that of simulation experiment.

\section{Working Principles of Metal Spinning and Main Factors Affecting the Dimensional Precision of Spinning Products}

The spinning machine is mainly comprised of spindle box, spindle, mandrel, main lathe bed, side lathe bed, cross-shaped sliding table, spinning roller, tail jacking device, heating system, cooling system, hydraulic system and electric system. Working principle of metal spinning: mount and clamp the workblank on the mandrel and make it rotate together with the mandrel; meanwhile, the spinning roller is used to rotate around the workblank and relative feed is achieved between the spinning roller and mandrel in order to make the workblank to be pressed and produce continuous local plastic deformation; in this way, the hollow rotational parts can be obtained ${ }^{[5-6]}$.

Both of the reduction of surface smoothness caused by too much feed amount and failure of profiling of workblank caused by too less feed amount will affect the molding quality of spinning products. And the spinning products are mainly influenced in two aspects: dimensional tolerance of diameter and surface roughness.

To ensure a stable feed rate of spinning roller can effectively improve the shaping quality of spinning products and the prerequisite to achieve this is to accurately determine the spinning force applied on the spinning roller in the process of spinning. At present, the evaluation of spinning force is conducted under the assumed condition that the metals are flowed axially in a stable and continuous manner; and this only applies to the stable power spinning process. As a result, this paper has put forward an algorithm which is based on the combined deformation of shearing and bending moment to calculate the spinning force.

\section{Algorithm of Spinning Force}

The following assumptions are required to be made before calculating the spinning forces of cone parts:

1) The workblank meets the required homogeneity and isotropy.

2) No negative volume is produced before and after the deformation of materials.

3) Neglecting the force of friction produced between the spinning roller and workblank.

4) When the workblank under the spinning roller is deformed instantaneously, other parts of the material will not be affected. rigid.

5) In the process of deformation, the equipment and tool system are regarded to be purely

6) The materials within the deformation zone are regarded in a plane deformation state.

Fig1 shows the deformation by power spinning; in this method, the assumption is made that during the deformation of cone parts by power spinning, the shear deformation in the direction of spin axis of mandrel shall be taken as the principal one; but meanwhile, the deformation of bending and anti-bending on the plane of $R Z$ and around axial $R$ is still existed.

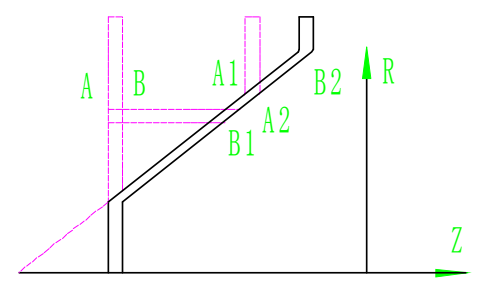

Fig1 Deformation of Spinning

Before the line element $A B$ that is on the workblank and with a distance of $R$ to the spin axis of mandrel reaches the position of $A_{1} B_{1}$, only the secondary deformation is produced; only after it reaches the position of $A_{1} B_{1}$ and contact with the spinning roller, the primary deformation will be produced. $n_{0}$ can be approximately calculated by the following equation: 


$$
n_{0}=\frac{r_{\rho} \operatorname{ctg} \alpha}{f}
$$

However, each deformation formed when the spinning roller passes through is the combined action of bending on the point $P$ and the anti-bending on the point $Q$. As a result, the steps required to calculate the stress are as follows: firstly calculate the total actual strain on the points of $P$ and $Q$; then calculate the total actual strain for each time when the spinning roller passes through; finally, calculate the total actual strain after the spinning roller passes through for $n_{0}$ times.

In cylindrical coordinate $\Delta \varepsilon_{R R}$, the strain components of all points are $\Delta \varepsilon_{R R}, \Delta \varepsilon_{\theta \theta}, \Delta \gamma_{Z Z}$, $\Delta \gamma_{\theta Z}$ and $\Delta \gamma_{R \theta}$. Here, $\Delta$ represents the differential strain and integral sign each time when the spinning roller passes through the line element. On the point $P$ where the line element starts to be bent:

$$
\begin{aligned}
& \Delta \varepsilon_{R R}=0 \\
& \Delta \gamma_{R \theta}=0
\end{aligned}
$$

The two tangential strain components are:

$$
\begin{aligned}
& \Delta \gamma_{R Z}=\Delta\left(\frac{\partial Z}{\partial R}\right) \\
& \Delta \gamma_{\theta Z}=\frac{1}{R} \Delta\left(\frac{\partial Z}{\partial \theta}\right)
\end{aligned}
$$

In the equation, $Z$ represents the displacement component of $Z$ axis.

It can be considered that the extension strain on the point $P$ is caused by bending. The bending strain $\Delta \varepsilon_{\alpha}$ can be approximately calculated by the formula as follows:

$$
\Delta \varepsilon_{\alpha} \approx \frac{h}{\rho}
$$

In the formula, $\rho$ represents the radius of curvature of arc on the section of $P Q ; h$ represents the distance between the point selected to the neutral layer in the direction of thickness. It can be considered approximately that:

$\Delta \varepsilon_{\theta \theta} \approx \Delta \varepsilon_{\alpha}$

According to the law of constant volume, the following equation can be obtained:

$\Delta \varepsilon_{R R}+\Delta \varepsilon_{\theta \theta}+\Delta \varepsilon_{z Z}=0$

Hence:

$\Delta \varepsilon_{\theta \theta}=-\Delta \varepsilon_{z Z} \approx \frac{h}{\rho}$

According to the strain energy theory, in each time when the spinning roller passes through, the total actual strain on the point $P, \Delta \overline{\varepsilon_{P}}$ can be expressed as follows:

$$
\Delta \overline{\varepsilon_{P}}=\frac{\sqrt{2}}{3} \sqrt{\left(\Delta \varepsilon_{E E}-\Delta \varepsilon_{\theta \theta}\right)^{2}} \begin{aligned}
& +\left(\Delta \varepsilon_{\theta \theta}-\Delta \varepsilon_{Z Z}\right)^{2} \\
& +\left(\Delta \varepsilon_{R R}-\Delta \varepsilon_{Z Z}\right) \\
& +\frac{3}{2}\left(\Delta \gamma_{R Z}^{2}+\Delta \gamma_{R \theta}^{2}+\Delta \gamma_{\theta Z}^{2}\right)
\end{aligned}
$$

In case that $c_{1}=\frac{2 \Delta \varepsilon}{\Delta \gamma_{R Z}}, \quad c_{2}=\frac{\Delta \gamma_{\theta Z}}{\Delta \gamma_{R Z}}$, then it can be obtained as follows:

$$
\Delta \overline{\varepsilon_{P}}=\frac{2}{\sqrt{3}}\left[\frac{1}{2} \int_{P} d \gamma_{R Z} \sqrt{1+c_{1}^{2}+c_{2}^{2}}\right]
$$

In case that the line element is bent to a reserved direction after reaching the point $Q$, the extension strain component $\Delta \varepsilon_{R R}$ and three shearing strain components are all zero; the actual strain on the point $Q, \Delta \overline{\varepsilon_{\theta}}$ can be expressed as follows:

$$
\Delta \overline{\varepsilon_{\theta}}=\frac{2}{\sqrt{3}} \Delta \varepsilon_{\alpha}
$$


The total strain of the line element selected in each time of passing through can be obtained:

$$
\Delta \overline{\varepsilon_{n}}=\Delta \overline{\varepsilon_{\alpha}}+\Delta \overline{\varepsilon_{Q}}=\frac{2}{\sqrt{3}}\left(\frac{1}{2} \int_{P} d \gamma_{R Z} \sqrt{1+c_{1}^{2}+c_{2}^{2}}+\Delta \varepsilon_{\alpha}\right)
$$

Suppose that $c_{1}$ and $c_{2}$ is kept unchanged, then:

$$
\Delta \overline{\varepsilon_{n}}=\frac{\Delta \gamma_{R Z}}{\sqrt{3}}\left(r_{1}+\sqrt{1+c_{1}^{2}+c_{2}^{2}}\right)
$$

And then all the actual strains $\left(\int d \bar{\varepsilon}\right)$ obtained when the spinning rollers are displaced from $A_{1} B_{1}$ to $A_{2} B_{2}$, i.e. when the spinning rollers get through the line elements for $n_{0}$ times can be calculated. Same as the method adopted to calculate $\Delta \overline{\varepsilon_{P}}$, the values of $\Delta \varepsilon_{x}, \Delta \gamma_{R Z}$ and $\Delta \gamma_{\theta Z}$ must also be determined first in order to calculate the value of $\int d \bar{\varepsilon} . \Delta \varepsilon_{\alpha}$ along the direction of thickness is a variable; its average value in the direction of thickness shall be selected in order to simplify calculation.

$$
\Delta \varepsilon_{\alpha}=\frac{t_{0}}{4 \rho_{n}}
$$

This neutral layer is coincided with the central layer; the average value of $\Delta \gamma_{\theta Z}$ can be calculated as follows:

$$
\Delta \gamma_{\theta Z}=\frac{1}{R_{n}} \Delta\left(\frac{\partial Z}{\partial \theta}\right)=\operatorname{tg} \delta
$$

The following can be determined approximately:

$$
\left\{\begin{array}{l}
\rho_{n} \approx \frac{\left(R_{n} \theta_{0}^{\prime}\right)^{2}}{2 m Z_{n}^{\prime}} \approx \frac{\left(R_{n} \theta_{0}\right)^{2}}{2 Z_{n}^{\prime}} \\
\operatorname{tg} \delta^{\prime} \approx \frac{2 m Z_{n}^{\prime}}{R_{n} \theta_{0}^{\prime}} \approx \frac{2 \sqrt{m} Z_{n}^{\prime}}{R_{n} \theta_{0}}
\end{array}\right.
$$

In this formula, $m$ represents coefficient and its value is between 0.5 and 1 . follows:

The strain produced when the spinning roller passes through for one time is expressed as

$$
\left\{\begin{array}{l}
\Delta \varepsilon_{\alpha}=\frac{t_{0} / 2}{\left(R_{n} \theta_{0}^{\prime}\right)^{2}} Z_{n}^{\prime} \\
\Delta \gamma_{\theta Z}=\frac{2 \sqrt{m}}{R_{n} \theta_{0}} Z_{n}^{\prime}
\end{array}\right.
$$

Suppose once again that the $c_{1}$ and $c_{2}$ will be kept to be constant not only when the spinning roller passes through for each time, but during the whole process of the deformation of line element, then the following equations can be obtained:

$$
\left\{\begin{array}{c}
c_{1}=\frac{\Delta \varepsilon_{\alpha}}{\Delta \gamma_{R Z} / 2}=\frac{\sum_{1}^{n_{0}}\left|\varepsilon_{\theta}\right|}{\sum_{1}^{n_{0}} \frac{\Delta \gamma_{\theta Z}}{2}} \\
c_{2}=\frac{\Delta \gamma_{\theta Z}}{\Delta \gamma_{R Z}}=\frac{\sum_{1}^{n_{1}} \Delta \gamma_{\theta Z}}{\sum_{1}^{n_{1}} \Delta \gamma_{R Z}}
\end{array}\right.
$$

It can be known from the geometrical relationship that 


$$
\left\{\begin{array}{l}
\sum_{1}^{n_{0}} Z_{n}^{\prime}=\gamma_{\rho}\left(\frac{1}{\sin \alpha}-1\right) \\
\sum_{1}^{n_{0}} \Delta \gamma_{R Z}=\operatorname{ctg} \alpha
\end{array}\right.
$$

The actual strain obtained when the spinning roller passes through for the $n_{0}$ times can be calculated as follows:

$$
\int d \bar{\varepsilon}=\sum_{1}^{n_{0}} \Delta \overline{\varepsilon_{n}} \quad=\frac{\operatorname{ctg} \alpha}{\sqrt{3}}\left(c_{1}+\sqrt{1+c_{1}^{2}+c_{2}^{2}}\right)
$$

Equation used to calculate the tangential force:

$P_{t}=t_{0} f \sin \alpha \int \sigma d \bar{\varepsilon}$

Equation used to calculate the radial force applied for the first halfway:

$P_{r}=P_{t} \frac{R_{c} \theta_{0}}{\sqrt{0.5} \cdot f \cos \alpha}\left(\frac{1-\sin \alpha}{\cos \alpha}+\frac{f}{2 \gamma_{\rho}}\right)$

Equation used to calculate the radial force applied for the second halfway:

$P_{r}=P_{t} \frac{R_{c} \theta_{0}}{f \cos \alpha}\left(\frac{1-\sin \alpha}{\cos \alpha}+\frac{f}{2 \gamma_{\rho}}\right)$

Equation used to calculate the axial force applied for the first halfway:

$P_{Z}=P_{t} \frac{R_{n} \theta_{0}}{\sqrt{0.5} \cdot f \cos \alpha}$

Equation used to calculate the axial force applied for the second halfway:

$P_{Z}=P_{t} \frac{R_{n} \theta_{0}}{f \cos \alpha}$

\section{Process Experiment}

With a view to verifying the accuracy of the algorithm, this paper utilizes the algorithm to build a model and carry out the simulation experiment of power spinning, in which the varying spinning forces and double spinning rollers are adopted. Then XYQK30-500 numerical control spinning machine from certain a research institution is utilized to conduct the spinning process experiment on the same workpiece; besides, the Electrometric Method is applied to measure the spinning force exerted on the spinning rollers and the experiment parameters involved are shown in Table 1.

Table 1 Experimental parameters

\begin{tabular}{|l|l|l|}
\hline Parameters & Numerical \\
\hline \multirow{3}{*}{ Blank } & Diameter & $76 \mathrm{~mm}$ \\
\cline { 2 - 3 } & Material & $\mathrm{Cu}$ \\
\cline { 2 - 3 } & Adjusting angle & $45^{\circ}$ \\
\hline Core mold & Fillet radius & $35.6 \mathrm{~mm}$ \\
\hline \multirow{2}{*}{ Feed } & Spindle speed & $116 \mathrm{r} / \mathrm{min}$ \\
\cline { 2 - 3 } & Feed speed & $0.94 \mathrm{~mm} / \mathrm{r}$ \\
\hline
\end{tabular}

The tangential force, radial force and axial force obtained after experiment are shown in Fig2, Fig3 and Fig 4 respectively. The green line in the figures represents the simulation value; red line represents the actually measured value; the blue line represents the value calculated by the new algorithm; and the yellow line represent the value calculated by the original algorithm. It can be seen from the result of process experiment that the value of spinning force calculated based on the combined deformation of shearing and bending moment is more inclined to approach the actually measured value. 


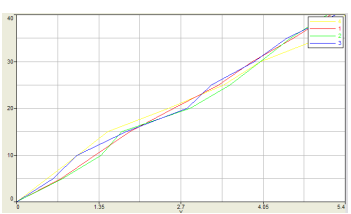

Fig 2 Shear spinning force

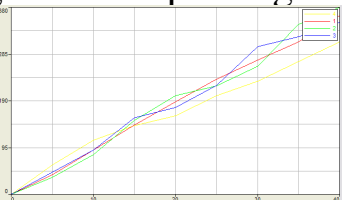

Fig 3 Radial spinning force

Fig 4 Axial spinning force

The spinning force calculated according to the new algorithm is utilized to set up again the process parameters and carry out the spinning process experiment of cone parts. The experiment parameters are the same with that shown in Table 1. Its experiment results are shown in Table 2.

Table2 Experimental results

\begin{tabular}{|l|l|l|}
\hline Check project & Required value $(\mathrm{mm})$ & Experimental values $(\mathrm{mm})$ \\
\hline $\begin{array}{l}\text { Wall thickness } \\
\text { difference }\end{array}$ & 0.02 & 0.02 \\
\hline 3/4Ellipticity & 0.03 & 0.02 \\
\hline 1/2Ellipticity & 0.02 & 0.01 \\
\hline 1/4Ellipticity & 0.01 & 0.008 \\
\hline 3/4Ellipticity & 0.06 & 0.02 \\
\hline 1/4Ellipticity & 0.04 & 0.015 \\
\hline Inside diameter & \pm 0.3 & \pm 0.04 \\
\hline
\end{tabular}

\section{Conclusion}

This paper has put forward an algorithm based on the combined deformation of shearing and bending moment to calculate the power spinning force of cone parts. It is verified by the Electrometric Method that this algorithm is accurate and valid. And this algorithm is based to conduct the process experiment; compared with the traditionally applied algorithm, the dimensional precision of diameter of spinning products calculated by this new algorithm has been improved to some extent.

\section{References}

[1] Zhao Yunhao, Li Yanli. Technoligy and application of spinning. Beijin: Machinery Industry Press, (2008)

[2] Matsunok. Recent Research and Development in Metal Forming in Japan, J, Mater, Process, Technol, Vol.2(1997),p.1-3

[3] Anon. Metal spinning in the auto motive industry,J, Sheet Metal Industries, Vol.5(1995),p.13-18

[4] Xue Kemin, Wang Zhen, Lu Yan. Elasto-plastic FEM Analysis and Experimental Study of Diametral Growth in Tube Spinning, J, Mater, Process, Technol,Vol.172-175(1997),p.69

[5] WANG Z T, XIE S S, JIN Q J. Elasto-plastic finite element analysis of hydrostatic extrusion with various mathematically contoured dies,J, Proceedings of the 24th International Machine Tool Design and Research Conference, eds, Davies BJ, Vol.52(1983),p.51-58

[6] HAYAMA, Hiroaki KUDO. Experimental Study of Tube Spinning,J, Bulletion of JSME. Vol.769-775(1979,p.167 